\title{
Relationship between General Health Indices and Sexual Dysfunction in Women Experiencing Spousal Abuse
}

\author{
Arezoo Shayan ${ }^{1}$, Masoumeh Kaviani ${ }^{2}$, Sara Azima ${ }^{2}$, Seyedeh Zahra Masoumi ${ }^{3}$, Sezaneh Haghpanah ${ }^{4}$, \\ Saeed Gholamzadeh ${ }^{5} \&$ Mohamad Zarenezhad $^{6}$ \\ ${ }^{1}$ Midwife, School of Nursing and Midwifery, Shiraz University of Medical Sciences, Shiraz, Iran \\ ${ }^{2}$ Department of Midwifery, School of Nursing and Midwifery, Shiraz University of Medical Sciences, \\ Shiraz, Iran \\ ${ }^{3}$ Research Center for Child and Maternity Care, Midwifery Department, School of Nursing And Midwifery, \\ Hamadan University of Medical Sciences, Hamadan, Iran \\ ${ }^{4}$ Community Medicine, Hematology Research Center, Shiraz University of Medical Science, Shiraz, Iran \\ ${ }^{5}$ Forensic Medicine, Shiraz, Iran \\ ${ }^{6}$ Legal Medicine Research Center, Legal Medicine Organization, Tehran, Iran \\ Correspondence: Masoumeh Kaviani, Department of Midwifery, School of Nursing and Midwifery, Shiraz \\ University of Medical Sciences, Shiraz, Iran. Tel: 98-91-7710-3797. E-mail: \\ kavianimaasumeh@yahoo.com
}

Received: January 22, 2016 Accepted: February 23, 2016 Online Published: March 10, 2016

doi:10.5539/gjhs.v8n10p275 URL: http://dx.doi.org/10.5539/gjhs.v8n10p275

\begin{abstract}
Background: Sexual behavior is among the complex dimensions of humans. Various factors are effective in sexual function. Sexual dysfunction affects people's mental health.

Objectives: The present study aims to determine the relationship between general health indices and sexual dysfunction among the women referring to Shiraz Forensic Medicine Center, Iran.

Methods: This cross-sectional, descriptive-analytical study was conducted on 197 women experiencing spousal abuse who had referred to Shiraz Forensic Medicine Center in 2014. The data were collected using demographic information questionnaire, a questionnaire assessing violence against women, general health questionnaire (GHQ-28), and female sexual function index (FSFI). The data were entered into SPSS statistical software and analyzed using t-test, Chi-square test, Pearson's correlation coefficient, and regression analysis.
\end{abstract}

Results: Based on the results, $91.9 \%$ of the participants had sexual dysfunction and $85.3 \%$ had mental disorders. Besides, a significant relationship was found between sexual dysfunction and somatic symptoms $(\mathrm{P}=0.027)$, anxiety and insomnia $(\mathrm{P}=0.004)$, as well as overall general health $(\mathrm{P}=0.041)$. Also, the results of Pearson's correlation test revealed significant associations between various dimensions of sexual dysfunction and general health.

Conclusion: According to the results, physical and mental health seems to play a critical role in determining sexual dysfunction. Thus, the identification and treatment of physical and mental disorders are recommended for the patients suffering from sexual dysfunction.

Keywords: general health, sexual dysfunction, spousal abuse, married women, forensic medicine

\section{Introduction}

Violence against women is the most serious result of social, economic, political, and cultural inequalities, which leads to mental and physical diseases, less participation of women in social activities, sadness, depression, emotional stress, sexual dysfunction, abortion, maternal hospitalization, delay in prenatal care, and risk of sexually transmitted infections (Latifi, Taghdisi, Eshagh, \& Azam, 2012).

Spousal abuse has many different forms and dimensions, ranging from physical misbehavior and psychological abuse to sexual misbehavior. However, it usually includes controlling behaviors such as isolating women from their families as well as friends and denying her access to different resources. Spousal abuse aims at controlling 
and violence is against woman in $90 \%$ of the cases (Garcia-Moreno \& Watts, 2011). Evidence has indicated that $66 \%$ of Iranian women have experienced violence at least once from the beginning of their marital life. Yet, the rate and type of domestic violence are significantly different in various provinces of Iran (Navroi \& Zeinali, 2012). Some studies have demonstrated a significant correlation between types of spousal abuse and female sexual dysfunction (Santos-Iglesias, Sierra, \& Vallejo-Medina, 2013).

Sexual response cycle includes several steps: desire, sexual excitement, stability, orgasm, and resting. In sexual desire and arousal disorder, while enjoying complete physical health, the person is not interested in sexual behavior and partnership (Berek, 2012; Hopkins et al., 2015). Sexual excitement disorder emerges with reduced vaginal wetness or dyspareunia. In physiological terms, orgasm refers to reaching the peak of sexual pleasure, before which the arousal step should be complete. The main problem of most patients is that they remain in the arousal step, could not reach the following step, and thus gradually lose their sexual desire. Women may be so sensitive to follow-up her own and her partner's responses and so concerned about failure that could not let her natural reflexes cause orgasm (Ramondetta, 2013). The prevalence of sexual dysfunction has been $52 \%$ in the U.S., 32\% in England, 34\% in Australia, 26\% in Japan, 28\% in China, and 37\% in Korea. The difference in the prevalence rates might be attributed to the demographic features of the research population and women's shamefulness in expressing their sexual problems (Lewis, 2012). According to the national investigation performed in Iran in $2005,31.5 \%$ of the women were suffering from sexual dysfunction. Although this rate is lower than some other countries, it indicates sexual problems as a major health problem among Iranian women (Ramazani, 2012). Patients with sexual disorders normally hide their problems and try to solve them at home. Therefore, these symptoms are not treated, eventually leading to chronic symptoms, mental disorders, and disappearance of tranquility from the couples' lives. Moreover, women suffering from sexual dysfunction have mood disorders, low self-confidence, introversion, feeling guilty, and depression, which might result in divorce (Takahashi, Inokuchi, Watanabe, Saito, \& Kai, 2011). Sexual satisfaction is defined as the judgment and analysis of every person about her/his own sexual relation which is considered enjoyable. Such satisfaction is influenced by mental factors, person's relationship with the sexual partner, environmental and cultural factors, diseases, and so on (Pourakbaran \& Yazdi, 2015). Considering the negative effects of sexual dysfunction and its resulting complications on the family and society and due to the fact that expression of sexual problems is not easy because of cultural issues, prevention and treatment of these disorders is the main responsibilities of the health team. Of course, this issue requires awareness about the prevalence of these disorders in the society so that the necessary measures can be taken to eliminate these problems (Takahashi, Inokuchi, Watanabe, Saito, \& Kai, 2011).

Objectives: Thus, the present study aims to assess the relationship between general health and female sexual dysfunction among the women referring to Shiraz Forensic Medicine Center, Iran.

\section{Methods}

This cross-sectional, descriptive-analytical study was conducted on the women experiencing spousal abuse who had referred to Shiraz Forensic Medicine Center from January to March 2014. The research population included all the women experiencing spousal abuse who had been referred by judicial authorities to Forensic Medicine Center and were willing to participate in the study. Considering the prevalence rate of sexual disorders based on the previous studies (31.5\%), $\alpha=0.05$, and accuracy of $6.5 \%$, the 197 -subject sample size was determined for the study. The subjects were selected through simple random sampling.

The inclusion criteria of the study were gaining sufficient scores in one of the dimensions of the questionnaire assessing violence against women $(\geq 16$ in physical violence, $\geq 11$ in physical violence, $\geq 3$ in sexual violence, and $\geq 2$ in economic violence), being willing to take part in the study, aging 16-45 years old, being able to read and write, living in Fars Province, not having severe physical injuries, and not being pessimistic. In case the individuals were not willing to continue their cooperation in the study or had not accurately completed the questionnaires, they were excluded from the study.

The data were collected using demographic information questionnaire, a questionnaire assessing violence against women, female sexual function index (FSFI), and general health questionnaire (GHQ-28).

Demographic information questionnaire included age, education, parity, number of children, history of drug abuse, history of addiction, housing status (tenant, own house, house of wife's parents, house of husband's parents, others), number of spouses, in-laws' interference, history of infertility, contraception methods, social status of wife's parents, husband's age, husband's occupation, husband's income level, husband's education, social status of husband's parent, husband's history of addiction, husband's history of imprisonment, and family's income level. 
The questionnaire assessing violence against women consisted of 32 items and 4 factors. The first (items 1-16), second (items 17-27), third (items 28-30), and fourth (items 31-32) factors dealt with mental, physical, sexual, and economic violence, respectively. The items could be responded through "Not at all", "Once", "Twice", and "More than twice" and, accordingly, received a score between 1 and 4. Thus, the scores of mental, physical, sexual, and economic violence could range from 16 to 64,11 to 44,3 to 12 , and 2 to 8 , respectively. Khaghani reported the Cronbach's alpha coefficient of this questionnaire as 0.97 , which was the basis of the present study (Saadati, 2012).

FSFI evaluated 6 dimensions of sexual function (sexual desire, arousal, orgasm, dyspareunia, lubrication, and sexual satisfaction) in the recent four weeks. In this scale, scores of $0-5$ were assigned to the items related to sexual desire, 1-5 to those related to arousal, lubrication, orgasm, and dyspareunia, and 0-5 to the items dealing with satisfaction. The total score of the index was also computed by summing up the scores of these 6 dimensions. Accordingly, higher scores represented better sexual functioning. In order to equiponderate the dimensions, the maximum score was considered 6 for each dimension and 36 for the whole scale. The reliability of the FSFI was confirmed through Cronbach's alpha $>0.70$ for all the dimensions. It should be noted that the cut-off point of 28 was considered for the diagnosis of sexual dysfunction (Ramondetta, 2013).

GHQ-28 which was introduced by Goldberg and Helyerand is a reliable instrument for determining individuals' mental disorders. This questionnaire includes 4 subscales, namely somatic symptoms (items 1-7), anxiety and insomnia (items 8-14), social dysfunction (items 15-21), and depression (items 22-28). The individuals receiving scores $>24$ were considered to have mental disorders. The reliability coefficients for all the subscales were above 0.70 (Saadati, 2012).

At first, the participants were provided with the questionnaire assessing violence against women. In case they obtained sufficient scores in any of the violence areas (the lowest score in one of the dimensions), they were given demographic information questionnaire, FSFI, and GHQ-28. This process continued until the sufficient number of samples was selected.

\subsection{Ethical Considerations}

At first, permission was gained from Vice-Chancellor of University for Research Affairs and the study was approved by Ethics Committee of the Shiraz University of medical sciense. Then, the research objectives were explained to the participants and they were reassured about the confidentiality of their information and that participation in the study was voluntary. Also, written informed consents for taking part in the study were obtained from all the participants.

\subsection{Statistical Analysis}

The data were entered into SPSS statistical software (v. 18) and analyzed using descriptive statistics, Chi-square, t-test, Pearson's correlation, and linear regression. Significance level was set at 5\%.

\section{Results}

This study was conducted on 197 women with complaints about spousal abuse and sexual dysfunction who had referred to Shiraz Forensic Medicine Center in 2014. According to the results, the mean age of the female and male participants was 30.42 and 35.15 years old, respectively, and most of the subjects had high school diplomas ( $43.7 \%$ of the women and $33 \%$ of the men). Besides, $83.2 \%$ of the women were homemakers.

Table 1. Means of general health dimensions in women experiencing spousal abuse

\begin{tabular}{lllll}
\hline Dimensions & Mean & SD & Maximum & Minimum \\
\hline Somatic symptoms (A) & 10.67 & 5.07 & 21 & 0 \\
Anxiety and insomnia (B) & 11.29 & 5.29 & 21 & 0 \\
Social dysfunction (C) & 11.10 & 4.82 & 21 & 1 \\
Depression (D) & 10.25 & 6.28 & 21 & 0 \\
Overall general health & 43.31 & 18.07 & 81 & 4 \\
\hline
\end{tabular}

The mean score of general health was 43.31. The highest mean score of general health was related to anxiety and insomnia, indicating the lower prevalence of these problems among the studied women. On the other hand, the lowest mean score of general health was related to depression, representing the higher prevalence of this disorder among the participants (Table 1). 
Table 2. Means of sexual function dimensions in women experiencing spousal abuse

\begin{tabular}{lllll}
\hline Dimensions & Mean & SD & Maximun & Minimum \\
\hline Sexual desire & 2.81 & 1.20 & 6 & 1.20 \\
Sexual arousal & 2.15 & 1.73 & 6 & 0 \\
Lubrication & 2.97 & 2.16 & 6 & 0 \\
Orgasm & 2.52 & 1.95 & 6 & 0 \\
Sexual satisfaction & 2.26 & 1.56 & 6 & 0.80 \\
Dyspareunia & 3.06 & 2.38 & 6 & 0 \\
Overall sexual function & 15.80 & 9.77 & 35.70 & 2 \\
\hline
\end{tabular}

The mean score of sexual dysfunction was 15.80 (Table 2). Considering the cut-off point, $85.3 \%$ of the subjects had mental disorder and $91.9 \%$ had sexual dysfunction.

Table 3. Relationship between overall sexual dysfunction and general health in women experiencing spousal abuse

\begin{tabular}{lll}
\hline Mental Health and Its Dimensions & Correlation Coefficient & P-value \\
\hline Somatic symptoms (A) & -0.157 & 0.027 \\
Anxiety and insomnia (B) & -0.205 & 0.004 \\
Social dysfunction (C) & -0.085 & 0.237 \\
Depression (D) & -0.019 & 0.787 \\
Overall mental health & -0.144 & 0.041 \\
\hline
\end{tabular}

The relationship between FSFI variables and general health was assessed using Pearson's correlation test. The results revealed a significant, negative, reverse relationship between sexual dysfunction and somatic symptoms $(\mathrm{P}=0.027)$, anxiety and insomnia $(\mathrm{P}=0.004)$, and overall general health $(\mathrm{P}=0.041)$. Thus, higher general health in the dimensions of somatic symptoms, anxiety and insomnia, and overall general health was accompanied by a reduction in sexual dysfunction. However, the linear relationships between sexual dysfunction and social dysfunction $(\mathrm{P}=0.237)$ and depression $(\mathrm{P}=0.787)$ were not statistically significant (Table 3$)$.

Table 4. Correlation between sexual dysfunction variables and mental health

\begin{tabular}{lllllll}
\hline & Variable & $\begin{array}{l}\text { Mental } \\
\text { Health }\end{array}$ & $\begin{array}{l}\text { Somatic } \\
\text { Sysptoms }\end{array}$ & $\begin{array}{l}\text { Anxiety } \\
\text { Insomnia }\end{array}$ & $\begin{array}{l}\text { Social Function } \\
\text { Depression }\end{array}$ & $\begin{array}{l}\text { Overall } \\
\text { Mental } \\
\text { Health }\end{array}$ \\
\hline \multirow{5}{*}{ Sexual } & Sexual desire & -0.001 & -0.00 & -0.076 & -0.455 & -0.008 \\
& Arousal & -0.066 & -0.015 & -0.230 & -0.796 & -0.102 \\
& Lubrication & -0.094 & -0.015 & -0.278 & -0.750 & -0.114 \\
& Orgasm & -0.023 & -0.004 & -0.165 & -0.734 & -0.050 \\
& Sexual & -0.026 & -0.014 & -0.128 & -0.144 & -0.014 \\
& Satisfaction & & -0.031 & -0.614 & -0.312 & -0.648 \\
& Dyspareunia & -0.262 & -0.047 & -0.787 & -0.041 \\
& Overall sexual & -0.027 & -0.004 & -0.237 & & \\
\hline
\end{tabular}

Pearson's correlation test was used to assess the relationship between sexual dysfunction and mental health dimensions. The results revealed significant correlations between somatic symptoms and sexual desire $(\mathrm{P}=-0.001)$, orgasm $(\mathrm{P}=-0.023)$, sexual satisfaction $(\mathrm{P}=-0.026)$, and overall sexual dysfunction $(\mathrm{P}=-0.027)$. Also, anxiety and insomnia were associated with all the dimensions of sexual function and overall sexual dysfunction (Table 4). However, no significant relationship was observed between social function and depression and any of the sexual dysfunction dimensions (Table 4). Furthermore, overall mental health was significantly associated 
with sexual desire $(\mathrm{P}=-0.008)$, orgasm $(\mathrm{P}=-0.050)$, and overall sexual dysfunction $(\mathrm{P}=-0.041)$, but not with other dimensions (Table 4). All of these relationships were negative and reverse; i.e. higher general health levels were accompanied by lower sexual dysfunction and vice versa.

\section{Discussion}

Women's health is one of the main priorities of the society's health. The present study aimed to determine the relationship between general health and sexual dysfunction among the women experiencing spousal abuse who had referred to Shiraz Forensic Medicine Center. According to the results, $8.1 \%$ of the participants had desirable sexual function, while the rest (91.9\%) complained about different degrees of sexual dysfunction. The results were in agreement with the findings reported by Mazinani et al. (2013), which represented the high prevalence of sexual dysfunction among women (Mazinani, Akbari Mehr, Kaskian, \& Kashanian, 2013). In the present study, $85.3 \%$ of the participants had mental disorders and a significant relationship was found between overall sexual dysfunction and somatic symptoms, anxiety and insomnia, and overall general health. Also, lower anxiety and insomnia were accompanied by lower sexual disorders. However, no significant association was observed between sexual dysfunction and other dimensions of general health (social function and depression). Fabre et al. (2012) also investigated mental health and proposed that sexual and mental disorders resulted from low mental health levels in a large number of women (Fabre \& Smith, 2012). Moreover, Montesi et al. (2013) examined the effects of anger and anxiety on men's and women's sexual desire and came to the conclusion that increased anger level led to decreased sexual desire in both men and women. Besides, a negative relationship was found between anxiety and sexual desire; i.e. as the level of anxiety increased, sexual desire decreased in both men and women (Montesi et al., 2013). Other studies have also demonstrated a significant relationship between mental health and sexual dysfunction (Berek, 2012; Lin, Juang, Wen, Liu, \& Hung, 2012; Kalmbach, Pillai, Kingsberg, $\&$ Ciesla, 2015). This was in agreement with the findings of the current work.

Our study's results revealed a significant association between somatic symptoms and sexual desire, orgasm, sexual satisfaction, and overall sexual dysfunction, implying that lower general health in somatic symptoms dimension was accompanied by increase in sexual desire, orgasmic, and sexual satisfaction disorders and overall sexual dysfunction. This finding was in line with the results of Eliason's (2014) study (Eliason, 2014). According to Sadock et al. (2007), the effective factors in orgasmic disorder included medical and general disorders, physical status, mental status, anxiety in sexual relationships and reaching orgasm, pelvic complaints such as lower abdominal pain, vaginal discharge, and itching, increased tension and irritability, fatigue, and fear from pregnancy (Sadock \& Sadock, 2011). The findings of the present study demonstrated that anxiety and insomnia were significantly related to the 6 dimensions of sexual dysfunction and overall sexual dysfunction. Accordingly, increase in anxiety and insomnia resulted in an increase in disorders in the whole sexual dysfunction dimensions as well as in overall sexual dysfunction. In general, different factors are associated with sexual desire disorders, including depression, anxiety, stress, abstinence from sexual relationship for a long time, hostility to the sexual partner, and marital conflicts. Moreover, lack of self-esteem, access to a suitable sexual partner, proper relationships with the sexual partner in non-sexual areas, age, and overall health status might decrease sexual desire (Sadock \& Sadock, 2011). Anxiety has been reported as an effective factor in sexual dysfunction. In this respect, a large number of studies have indicated that, when such individuals are faced with sexual stimulants, they get worried about their sexual performance and are distracted from sexual stimulants, which could lead to a decrease in their sexual arousal (Montesi et al., 2013). The present study's results showed no significant relationships between sexual dysfunction dimensions and depression and social function. In contrast, Lai et al. disclosed that reduced sexual desire and orgasm were highly prevalent among depressed women. Controlling depression symptoms can improve individuals' satisfaction and sexual function, which seems to support the importance of psychological issues in the incidence of sexual disorders (Takahashi, Inokuchi, Watanabe, Saito, \& Kai, 2011). In general, human's sexual satisfaction is mostly a product of mind rather than body and depression, anger, anxiety, and fear can interfere with the ability to enjoy sexual activities (Atlantis \& Sullivan, 2012). Suffering from organic and physical diseases could affect sexual activity and satisfaction. Non-organic factors, such as barriers related to social-religious beliefs, sexual injuries, and bad sexual experiences, can have an impact on normal sexual function as well. Depression, mental pressure, chronic diseases, social function, infertility, pregnancy, inappropriate relationship with one's spouse, delivery, social status, lifestyle, and marriage characteristics could also affect individuals' sexual satisfaction(Christensen et al., 2011). The findings of the current study demonstrated a significant correlation between overall general health and sexual desire, orgasm, and overall sexual dysfunction. Accordingly, as the overall general health decreased, sexual desire disorder, orgasmic disorder, and overall sexual dysfunction increased. Similarly, Ghaedi et al. (2013) reported a significant relationship between the symptoms of mental disorders and sexual disorders (Ghaedi, 2013). One of the 
limitations of the present study was the large number of questionnaires and some participants' lack of cooperation due to cultural issues and Small sample size.

\section{Conclusion}

Based on the findings of the current study, individuals' general health seems to be associated with sexual dysfunction and their mental health can, to some extent, predict sexual dysfunction. Ability to have appropriate sexual relationships requires having skills which are both trainable and learnable. Increasing couples' skills in the aforementioned dimensions can, in turn, increase their marital adjustment, satisfaction, and general health. Hence, training and consultation programs regarding sexual issues and improvement of mental health are recommended to be conducted for couples in order to increase their marital satisfaction and enhance families' stability. In addition, provision of psychological services in gynecology and other related centers can play a critical role in the prevention and treatment of these disorders.

\section{Acknowledgments}

This article is a part of an MSc thesis supported by Shiraz University of Medical Sciences. We would like to thank Vic-Chancellor of Education as well as Vice-Chancellor of Research and Technology of Shiraz University of Medical Sciences for financial support of this study. The authors declare that they have no conflicts of interest.

\section{Conflict of Interest}

The authors declare that there is no conflict of interests regarding the publication of this paper.

\section{References}

Atlantis, E., \& Sullivan, T. (2012). Bidirectional association between depression and sexual dysfunction: A systematic review and meta-analysis. The Journal of Sexual Medicine, 9(6), 1497-1507. http://dx.doi.org/10. 1111/j.1743-6109.2012.02709.x

Berek, J. S. (2012). Berek \& Novak Gynocology: Lippncott Williams \& Wilkins. Phiadelphia.

Christensen, B. S. et al. (2011). Associations between physical and mental health problems and sexual dysfunctions in sexually active Danes. The Journal of Sexual Medicine, 8(7), 1890-1902. http://dx.doi.org/10.1111/j.1743-6109.2010.02145.x

Eliason, M. J. (2014). Chronic physical health problems in sexual minority women: Review of the literature. LGBT health, 1(4), 259-268. http://dx.doi.org/10.1089/lgbt.2014.0026

Fabre, L. F., \& Smith, L. C. (2012). The effect of major depression on sexual function in women. The Journal of Sexual Medicine, 9(1), 231-239. http://dx.doi.org/10.1111/j.1743-6109.2011.02445.x

Garcia-Moreno, C., \& Watts, C. (2011). Violence against women: An urgent public health priority. Bulletin of the World Health Organization, 89(1), 2. http://dx.doi.org/10.2471/BLT.10.085217

Ghaedi, G. (2013). Examine the relationship between psychological disorders and sexual dysfunction. Daneshvar, 20(104), 9-18.

Hopkins, T. G., Stavraka, C., Gabra, H., Fallowfield, L., Hood, C., \& Blagden, S. (2015). Sexual activity and functioning in ovarian cancer survivors: An internet-based evaluation. Climacteric, 18(1), 94-98. http://dx.doi.org/10.3109/13697137.2014.929104

Kalmbach, D. A., Pillai, V., Kingsberg, S. A., \& Ciesla, J. A. (2015). The transaction between depression and anxiety symptoms and sexual functioning: A prospective study of premenopausal, healthy women. Archives of Sexual Behavior, 44(6), 1635-1649. http://dx.doi.org/10.1007/s10508-014-0381-4

Latifi, M., Taghdisi, M. H., Eshagh, A. M., \& Azam, K. (2012). Effect of Life Skills Training on Self-Esteem and Decreasing Violence against Women.

Lewis, R. W. (2012). Epidemiology of sexual dysfunction in Asia compared to the rest of the world. Asian $J$ Androl, 13(1), 152-158. http://dx.doi.org/10.1038/aja.2010.108

Lin, C. F., Juang, Y. Y., Wen, J. K., Liu, C. Y., \& Hung, C. I. (2012). Correlations between sexual dysfunction, depression, anxiety, and somatic symptoms among patients with major depressive disorder. Chang Gung Medical Journal, 35(4), 323-331.

Mazinani, R., Akbari, M., Mandana, K., Adis, \& Kashanian, M. (2013). Evaluation of prevalence of sexual dysfunctions and its related factors in women. Razi Journal of Medical Sciences, 19(105).

Montesi, J. L., Conner, B. T., Gordon, E. A., Fauber, R. L., Kim, K. H., \& Heimberg, R. G. (2013). On the 
relationship among social anxiety, intimacy, sexual communication, and sexual satisfaction in young couples. Archives of Sexual Behavior, 42(1), 81-91. http://dx.doi.org/10.1007/s10508-012-9929-3

Pourakbaran, E., \& Yazdi, S. A. A. (2015). A study of sexual functioning and marital satisfaction in women with and without history of labor. Journal of Fundamentals of Mental Health, 17(4), 202-208.

Ramazani, F., et al. (2012). Sexual dysfunction and its influencing factors: Population-based study among women living in urban areas, four Provinces. Payesh, 11(6), 869-875.

Ramondetta, L. (2013). What is the appropriate approach to treating women with incurable cervical cancer? $J$ Natl Compr Canc Netw, 11(3), 348-355.

Saadati, M. (2012). Domestic violence, mental health threat. Social Security Studies, (24), 61-90.

Sadock, B. J., \& Sadock, V. A. (2011). Kaplan and Sadock's synopsis of psychiatry: Behavioral sciences/clinical psychiatry. Lippincott Williams \& Wilkins.

Santos-Iglesias, P., Sierra, J. C., \& Vallejo-Medina, P. (2013). Predictors of sexual assertiveness: The role of sexual desire, arousal, attitudes, and partner abuse. Archives of sexual behavior. Archives of Sexual Behavior, 42(6), 1043-1052. http://dx.doi.org/10.1007/s10508-012-9998-3

Sotodeh Navroi, O., \& Zeinali, S. H. (2012). Relationship between spouse abuse, social support and perceived stress in women with addicted and non-addicted husbands in Rasht city. Holistic Nursing Practice, 22(68), 25-32.

Takahashi, M., Inokuchi, T., Watanabe, C., Saito, T., \& Kai, I. (2011). The female sexual function index (FSFI): Development of a Japanese version. The Journal of Sexual Medicine, 8(8), 2246-2254. http://dx.doi.org/10. 1111/j.1743-6109.2011.02267.x

\section{Copyrights}

Copyright for this article is retained by the author(s), with first publication rights granted to the journal.

This is an open-access article distributed under the terms and conditions of the Creative Commons Attribution license (http://creativecommons.org/licenses/by/3.0/). 